\title{
In Search of a Map: using Program Slicing to Discover Potential Parallelism in Recursive Functions
}

\author{
Adam D. Barwell and Kevin Hammond \\ School of Computer Science \\ University of St Andrews \\ \{adb23,kh8\}@st-andrews.ac.uk
}

\begin{abstract}
Recursion schemes, such as the well-known map, can be used as loci of potential parallelism, where, e.g., map is replaced with an equivalent parallel implementation. This paper formalises a novel technique, using program slicing, that automatically and statically identifies computations in recursive functions that can be lifted out of the function and potentially performed in parallel. We define a new program slicing algorithm, built a prototype implementation, and demonstrated its use on 12 Haskell examples, including benchmarks from the NoFib suite and functions from the standard Haskell Prelude. In all cases, we obtain the expected results in terms of finding potential parallelism. Moreover, we have tested our prototype against synthetic benchmarks, demonstrating our prototype has quadratic time complexity. For the NoFib benchmark examples we demonstrate that relative parallel speedups can be obtained (up to $32.93 \times$ the sequential performance on 56 hyperthreaded cores).
\end{abstract}

ACM Reference format:

Adam D. Barwell and Kevin Hammond. . In Search of a Map: using Program Slicing to Discover

Potential Parallelism in Recursive Functions. In Proceedings of Submitted to FHPC, Oxford, UK, 2017, 12 pages.

DOI: $10.1145 /$ nnnnnnn.nnnnnnn

\section{Introduction}

Traditional parallelisation techniques commonly consist of lowlevel primitives and libraries. They require the programmer to manually introduce and manage complex constructs, e.g. threads, communication, and synchronisation. This results in a tedious, difficult, and often error-prone process [22]. Structured parallelisation techniques, such as algorithmic skeletons, instead present instances of high-level composable patterns to the programmer [11]. They allow the parallel structure of a program to be expressed as an instance of a pattern, while abstracting over low-level primitives and libraries. Despite the simplified interface, introducing skeletons can still be a non-trivial task, particularly with large legacy applications [3]. This often requires significant knowledge of the code base, language, and parallelism itself [6]. One common problem arises when control or data dependencies inhibit, or obstruct, the introduction of parallelism. This paper introduces a new technique to minimise such obstructive dependencies using program shaping, so enabling the automatic introduction of algorithmic skeletons.

Recursion schemes describe how data structures can be traversed or constructed [31]. In functional languages, recursion schemes are often implemented as higher-order functions such as map or foldr. Moreover, since algorithmic skeletons can be seen as parallel implementations of higher-order functions, it is possible to use

Submitted to FHPC, Oxford, UK

978-x-xxxx-xxxx-x/YY/MM... \$15.00

DOI: $10.1145 /$ nnnnnnn.nnnnnnn recursion schemes as loci of potential parallelism [7]. For example, a call to a standard map can be replaced with a call to an equivalent parallel implementation, provided that all mapped computations are independent [35]. Unfortunately, there is no guarantee that higher-order functions will be used in all possible instances by the programmer, or that the best higher-order function will be used. It follows that if these higher-order functions can be otherwise discovered automatically, then more options for introducing parallelism become available to the programmer.

In [4], we presented a high-level description of how program slicing might be used to discover map operations in recursive functions, and demonstrated that those map operations can be used as loci of potential parallelism to produce relative speedups. In this paper, we give a more formal presentation of the approach presented in [4], placing greater emphasis on our classification technique, and apply that technique to a wider range of examples. Moreover, we define a novel program slicing [34] algorithm to inspect how the values of variables change between recursive calls. As a basis for our analysis, we define a strict, uncurried, and higher-order expression language. We have implemented our approach in Erlang, and demonstrated its use on 12 examples, including ones derived from the NoFib benchmark suite [32] and functions from the Haskell Prelude. Our prototype correctly discovers all possible mappable operations in our examples. Experimental results on some synthetic benchmarks suggest that our prototype has quadratic time behaviour. Moreover, we show that relative speedups are possible through the (semi-)automatic introduction of parallel map operations. We achieve a maximum of $32.93 \times$ speedup on a 56-core hyperthreaded experimental testbed machine.

\section{Illustrative Example}

We illustrate our approach using an example from the the NoFib suite, sumeuler, that calculates Euler's totient function for a list of integers and sums the results.

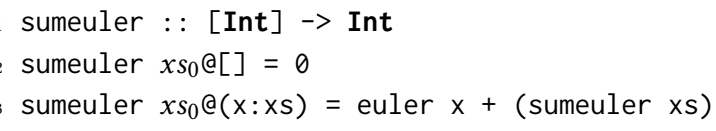

Here, we have unfolded (in the transformational sense) the definition given in the NoFib suite for demonstration purposes. Since this definition of sumeuler is explicitly recursive, parallelising the function definition requires the programmer to know how sumeuler traverses its arguments. This includes understanding how the computations performed on the (elements of the) input list affect: $i$ ) how the input is traversed; and ii) the result of sumeuler itself. To take advantage of recursion schemes as loci of potential parallelism, the above definition of sumeuler can be rewritten as an instance of a composition of a foldr and a map, 


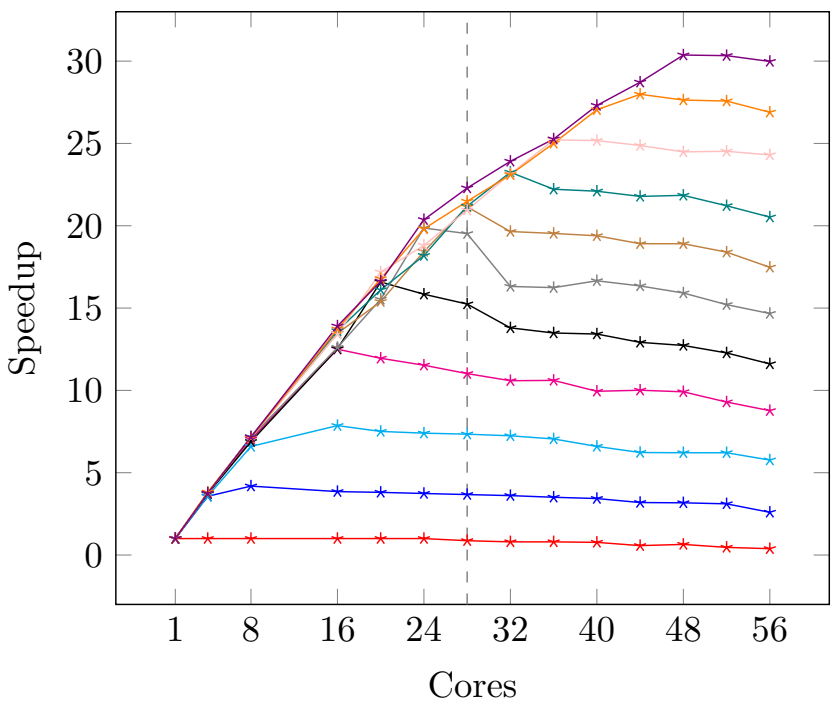

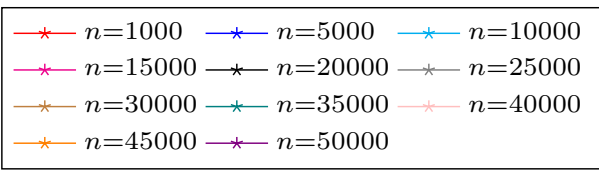

Figure 1. sumeuler, speedups on corryvreckan using reported mutator (MUT) time, dashed line shows extent of physical cores.

1 sumeuler : : [Int] $\rightarrow$ Int

2 sumeuler $x s_{0}=$ foldr $(+) \oslash$ (map euler $\left.x s_{0}\right)$

This simplifies the introduction of parallelism. Multiple parallelisations of, e.g., map are possible. We can use, for example, parList from the Haskell Strategies library [37], parMap from the Par Monad [30], or GPUs via the Accelerate library [10]. The parallel map skeleton can be used to safely plug-replace any standard use of map provided that all the mapped computations are independent. In the case of sumeuler, for example, we might use the standard Haskell Strategies library,

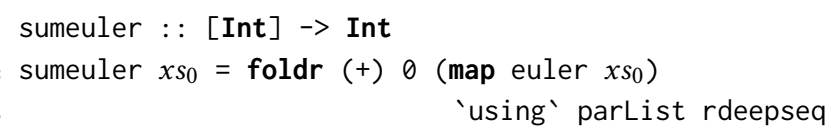

where the parallel map skeleton, parList, is parameterised with rdeepseq, a nested strategy that forces each element of the result to be fully evaluated. By cleanly separating the functional definition of the program from its evaluation strategy, it is easy to introduce alternative parallelisations, while still ensuring that the parallel version is functionally equivalent to the original definition. For example, the parallelisation can be further tuned to improve speedups, perhaps by chunking $x s_{0}$ [7], and by taking advantage of the associativity of $(+)$ to sum the individual chunks before returning the sum of all the chunks.

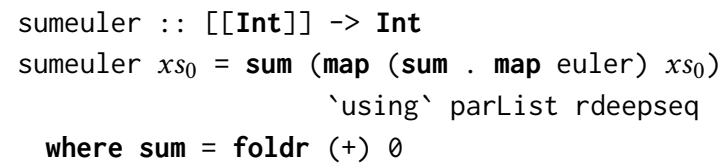

Fig. 1 shows the raw speedups that we obtain for this program for the interval $[1, n]$ for varying sizes of $n$ on our 56-core hyperthreaded experimental machine, corryvreckan. We obtain a maximum speedup of 30.50 for $n=50000$ on 48 hyperthreaded parallel cores.

In order to take advantage of skeletons, recursion schemes must be used explicitly. When recursion schemes are implicit, e.g. as in sumeuler, they must first be discovered either manually or automatically. To automatically discover the computations that may be performed as part of a map, we inspect the application expressions that are not recursive calls, hereafter termed operations, in the definition of sumeuler. Whether an operation may be lifted into a map depends on two things: $i$ ) how the arguments to the recursive function differ between the stages of the recursion; and ii) the structure of the arguments in the operation. We determine how the values of arguments change between recursive calls using program slicing. This is a technique that is used to extract all the program statements that, depending on the algorithm used, may influence, or may be influenced by, a given statement from the same program, called the slicing criterion [34]. A slice is often calculated by inspecting the control and data dependencies of a program. Slicing the explicitly recursive definition of sumeuler, taking $x$ as the criterion, produces:

1 sumeuler $x s_{0}\left(\mathrm{x}:_{-}\right)=$euler $\mathrm{x}+$ undefined

Both the empty-list clause and the recursive call, (sumeuler xs), are removed because they are not influenced by the value of $x$. Slicing might therefore be thought of as a narrowing of focus on only those parts of the program or expression that are of interest, and with all the irrelevant parts stripped away [4]. For the purposes of our approach, we can further narrow the slice to highlight how the values of the arguments change between recursive calls. Our slice only needs to be a set of variables, where inclusion in the set indicates use, and an annotated inclusion in the set indicates update. For example, the slice of sumeuler, with criterion $x s_{0}$, $\{x, x s\}$, indicates that both $x$ and $x$ s are used; in (euler $x$ ) and (sumeuler $\mathrm{xs}$ ) respectively. The slice also indicates that $x s_{0}$ itself is not considered to be updated. We consider a variable to be updated, when its value is significantly changed. For a list, we do not consider recursively traversing a list in this way a significant change. We formalise this intuition in Defns. 4.1 and 4.3.

Given such a slice, we now need to decide whether that argument can be used within a map, where each use is independent of all other uses. If the argument is used and not updated, e.g. as with $x s_{0}$ in sumeuler, then it can also occur safely in a map operation. Conversely, if the argument is updated but not used, e.g. $\mathrm{x}$ in,

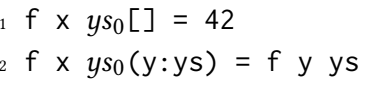

then it can similarly be updated independently of each input. In either of these cases, we consider the argument to be clean. However, when an argument is both used and updated, then it indicates that the usage and update of the variable is not independent of the other stages of the recursion. Any operation in which an argument that is both used and updated cannot be safely lifted into a map. We therefore consider such an argument to be tainted. In sumeuler, $x s_{0}$ is the only argument, and it is used but not updated. It is therefore classified as clean, along with its case-defined variables, $\mathrm{x}$ and $\mathrm{xs}$. 


$$
\begin{aligned}
& \text { BOOL }_{1} \overline{\Gamma \vdash \text { true }: \text { bool }} \quad \text { BOOL }_{2} \overline{\Gamma \vdash \text { false }: \text { bool }} \quad \text { INT } \overline{\Gamma \vdash \mathbb{Z}: \text { int }} \quad \text { var } \overline{\Gamma \cup\{x: \tau\} \vdash x: \tau} \quad \operatorname{LIST}_{1} \overline{\Gamma \vdash \text { nil }_{\tau}: \text { list } \tau} \\
& \Gamma \vdash x s: \text { list } \tau_{1} \quad \Gamma \vdash y: \tau_{1} \quad \Gamma \vdash y s: \text { list } \tau_{1} \quad \Gamma \vdash e_{1}: \tau_{2} \\
& \operatorname{LisT}_{2} \frac{\Gamma \vdash e_{1}: \tau \quad \Gamma \vdash e_{2}: \text { list } \tau}{\Gamma \vdash \operatorname{cons}_{\tau} e_{1} e_{2}: \text { list } \tau} \quad \operatorname{CASE} \frac{\Gamma \cup\left\{y: \tau_{1}, y s: \text { list } \tau_{1}\right\} \vdash e_{2}: \tau_{2}}{\Gamma \vdash \operatorname{case} x s \text { of } \operatorname{nil}_{\tau_{1}} \rightarrow e_{1}, \operatorname{cons}_{\tau_{1}}(y, y s) \rightarrow e_{2}: \tau_{2}} \quad \operatorname{APp} \frac{\Gamma \vdash e_{0}: \vec{\tau} \rightarrow \tau_{m} \quad \Gamma \vdash \vec{e}: \vec{\tau}}{\Gamma \vdash e_{0} \vec{e}: \tau_{m}} \\
& \mathrm{FUN} \frac{\Gamma \cup\{\vec{x}: \vec{\tau}\} \vdash e: \tau_{m}}{\Gamma \vdash \lambda \vec{x} \rightarrow e: \vec{\tau} \rightarrow \tau_{m}} \quad \operatorname{FIx} \frac{\Gamma \vdash e:\left(\left(\left(\tau_{2}, \ldots, \tau_{n}\right) \rightarrow \tau_{m}\right), \tau_{2}, \ldots, \tau_{n}\right) \rightarrow \tau_{m}}{\Gamma \vdash \operatorname{fix} e:\left(\tau_{2}, \ldots, \tau_{n}\right) \rightarrow \tau_{m}}
\end{aligned}
$$

Figure 2. Typing judgements for $E$, determining simple types in $T$.

Having determined whether each argument in our recursive function is clean or tainted, we can then determine for each operation in the same recursive function whether that operation is independent of successive recursive calls. Such an operation is classified as unobstructive when none of its arguments use a tainted variable or include a recursive call. For example, sumeuler has two operations:

1. (euler $x$ ); and

2. $(($ euler $x)+($ sumeuler $x s))$.

Since $\mathrm{x}$ is classified as clean, (euler $\mathrm{x}$ ) is classified to be unobstructive. Conversely, since the second argument to $(+)$ in the second operation is a recursive call, the addition operation is classified as obstructive. We can therefore lift (euler $\mathrm{x}$ ) into a map operation, perhaps by refactoring the original source:

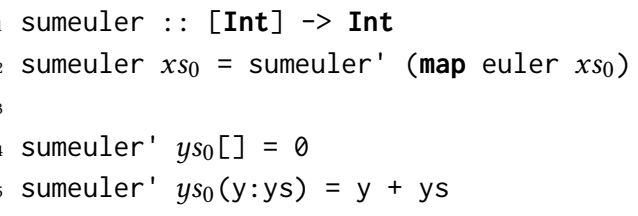

Here sumeuler' is functionally equivalent to sum, so our final form is:

1 sumeuler : : [Int] $\rightarrow$ Int

2 sumeuler $x s_{0}=$ sum $\left(\right.$ map euler $\left.x s_{0}\right)$

This can then be easily parallelised by using a parallel map skeleton, as seen above.

\section{Preliminaries and Assumptions}

We illustrate our approach over the simple expression language, $E$.

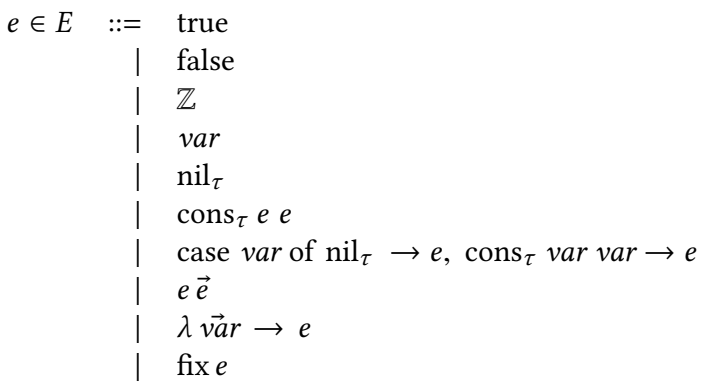

$E$ is a simple, strict, functional language. Its terms form a common subset of functional languages: boolean constants, true and false; integer constants, $z \in \mathbb{Z}$; variables, var; list constructors, nil $_{\tau}$ and $\operatorname{cons}_{\tau}$ e e ; case discrimination on lists, case var of nil $\operatorname{li}_{\tau} \rightarrow$ $e, \operatorname{cons}_{\tau}$ var var $\rightarrow e$; function application, $e \vec{e}$; lambda expressions, $\lambda \overrightarrow{v a r} \rightarrow e$; and fixpoints, fix $e$. Constructors are restricted here to cons-lists for simplicity and clarity of presentation, but the

$$
\begin{aligned}
& \text { def sumeuler = fix } \lambda\left(f, x s_{0}\right) \rightarrow \\
& \qquad \begin{array}{l}
\text { case } x s_{0} \text { of } \\
\operatorname{nil}_{\pi} \rightarrow 0, \\
\operatorname{cons}_{\pi}(x, x s) \rightarrow \text { plus }(\text { euler }(x), f(x s))
\end{array}
\end{aligned}
$$

Figure 3. Definition of sumeuler in $E$.

approach is extensible to other types, given a definition of variable update (Def. 4.1) for that type. Similarly, the approach can be extended to arbitrary types, given that a definition of variable update can be derived for arbitrary constructors. Vector notation, e.g. $\vec{e}$, refers to a non-empty tuple: $\vec{e} \equiv\left(e_{1}, \ldots, e_{n}\right), n \geq 1$. Tuples are purely meta-syntactic, and are used for clarity of notation. In order to simplify our presentation, list constructors and case discriminators are annotated with the (monomorphic) type of the list elements, $\tau$. The corresponding type language, $T$, is shown below.

$$
\begin{array}{rll}
\tau \in T \quad:= & \text { bool } \\
& \mid \text { int } \\
& \text { list } \tau \\
& \vec{\tau} \rightarrow \tau
\end{array}
$$

The typing judgements in Fig. 2 then determine the well-formedness of expressions in $E$ with regard to their monomorphic types in $T$. A statement, $s \in S$, is an assignment.

$$
\begin{array}{rlrl}
s \in S::= & \text { def } v a r & =\lambda \overrightarrow{v a r} \rightarrow e \\
& \mid \quad \text { def } \text { var }=\text { fix } e
\end{array}
$$

Statements appear only at the top level of a program. A variable may be bound either to a function application or to a fixpoint expression. These bindings are then in scope for the duration of the program. A program $p \in P$ is a series of statements.

$$
\begin{aligned}
& p \in P \quad::=s \\
& \text { | } s ; p
\end{aligned}
$$

$P$ can be thought of as an intermediate representation to which, e.g., Haskell or Erlang are compiled, similar to Core Haskell. For example, the Haskell definition of sumeuler,

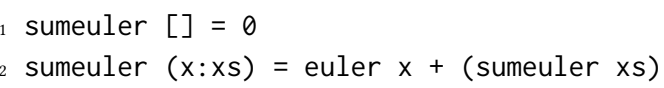

can be translated into the term in $P$ given in Fig. 3. In this paper, we will use Haskell syntax for our examples in order to improve readability. All our examples can be translated into $P$ following a similar principle to the above example. Our approach will inspect only the code provided and does not presume to predict possible compiler optimisations, e.g. fusions or worker-wrapper transformations. As an intermediate representation, these techniques can be applied after, or prior to, the application of our approach. 
Where pattern matching is used, we will use as-patterns to indicate the implicit list variables; e.g.

1 sumeuler $x s_{0} @[]=0$

2 sumeuler $x s_{0} @(\mathrm{x}: \mathrm{xs})=$ euler $\mathrm{x}+($ sumeuler $\mathrm{xs})$

For clarity, all the variables in our examples will be consistent across function clauses. All variables are assumed to be unique under $\alpha$-conversion, at both the statement and expression levels. Type environments, $\Gamma$, are defined to be a set of bindings of variables to types:

$$
\Gamma \in\{\text { var }: T\}
$$

As usual, all values in the domain of $\Gamma$ are assumed to be unique, and $\Gamma(x)$ denotes the type $\tau$ of a variable $x$ in $\Gamma$, such that $\exists \tau \in$ $T,(x: \tau) \in \Gamma$. For a given program $p$, the program environment, $\Gamma_{p}$, contains all the variables in $p$.

Definition 3.1 (Program Environment, $\Gamma_{p}$ ). Given some program $p$ and the set of variables $X \subseteq$ var that occur (either free or bound) in $p$, we define an environment $\Gamma_{p}$ to be a set such that $\forall x \in X, \exists \tau \in$ $T, x: \tau \in \Gamma_{p}$

The above sumeuler definition, for example, has the $\Gamma_{p}$ :

$$
\begin{aligned}
& \Gamma_{p}=\left\{x s_{0}: \text { list int }, x: \text { int }, x s: \text { list int },\right. \\
& f:(\text { list int }) \rightarrow \text { int }, \text { euler }:(\text { int }) \rightarrow \text { int }, \ldots\}
\end{aligned}
$$

We omit the variables and types of euler for clarity. For the rest of this paper, we will assume that for all given variables $x \in \Gamma_{p}$.

It is useful to define the notion of subexpressions in $E$, since subexpressions are a key element in both slicing and classification definitions.

Definition 3.2 (Subexpression). Given two expressions $e, e^{\prime}$, say that $e^{\prime}$ is a subexpression of $e\left(\right.$ denoted $\left.e^{\prime} \ll e\right)$ when

$$
\begin{gathered}
\frac{e^{\prime}=e}{e^{\prime} \ll e} \frac{e^{\prime} \ll e_{1} \vee e^{\prime} \ll e_{2}}{e^{\prime} \ll \operatorname{cons}_{\tau} e_{1} e_{2}} \\
\frac{e^{\prime} \ll e_{1} \vee e^{\prime} \ll e_{2}}{e^{\prime} \ll \operatorname{case} x \text { of } \operatorname{nil}_{\tau} \rightarrow e_{1}, \operatorname{cons}_{\tau} x^{\prime} x^{\prime \prime} \rightarrow e_{2}} \\
\frac{\exists i \in[0, n], e^{\prime} \ll e_{i}}{e^{\prime} \ll e_{0} \vec{e}} \quad \frac{e^{\prime} \ll e}{e^{\prime} \ll \lambda \vec{x} \rightarrow e} \quad \frac{e^{\prime} \ll e}{e^{\prime} \ll \text { fix } e}
\end{gathered}
$$

Subexpressions form a partial order relation.

We will refer to any application that is a subexpression of a fixpoint and that is not a recursive call as an operation. For example, the fixpoint expression in Fig. 3, has two operations: plus and euler. The application subexpression, $f(x s)$ is not an operation because it is a recursive call.

Functions are introduced using a $\lambda$-expression. They are always pure, are uncurried, and are never partially applied. They may, however, be higher-order. Any recursive (function) definitions are always introduced using an explicit fixpoint expression, e.g.:

$$
\operatorname{fix}(\lambda(f, x s) \rightarrow f(x s)) \text {. }
$$

The form of recursion is not otherwise restricted; general recursive forms are allowed, for example.

Lists are defined to be an ordered collection of elements, where those elements are accessed via case-expressions. As shown by the typing rules of Fig. 3, case discrimination is restricted to lists of some type $\tau$. We assume the existence of a built-in functions (e.g. if, eq, plus) for discrimination and operations on integers and booleans. Case-expressions can be extended to other types, given an additional check on the type of the discriminated variable in the relevant definitions. We limit case-expressions here to simplify our presentation. In the non-nil branch of a case-expression, new variables are bound respectively to the first element in the list (i.e. the head) and to the remainder of the list (i.e. the tail). A corresponding Reachability Relation is defined for each case-expression.

Definition 3.3 (Reachability Relation). Given a program $p$, a program environment, $\Gamma_{p}$, and a case-expression in $p, e=$ case $x s_{0}$ of $\operatorname{nil}_{\tau} \rightarrow e_{1}, \operatorname{cons}_{\tau} x s x s \rightarrow e_{2}$, we say that $x \triangleleft_{p} x s_{0}$ and $x s \triangleleft_{p} x s_{0}$. The transitive closure of the reachability relation is defined such that $z \triangleleft_{p}^{+} x s_{0}$ when $\exists y, z \triangleleft_{p}^{+} y \wedge y \triangleleft_{p} x s_{0}$. The reflexive-transitive closure of the reachability relation is defined such that $y \triangleleft_{p}^{*} x s_{0}$ when $\exists y, y \triangleleft_{p}^{+} x \vee y=x s_{0}$.

For example, $x \triangleleft_{p} x s_{0}, x s \triangleleft_{p} x s_{0}$, and $x s_{0} \triangleleft_{p}^{*} x s_{0}$ all hold for the case expression in sumeuler. The Reachability Relation will be used to calculate the program slice for a given expression and variable. It is also useful to know when a cons-expression reconstructs $x s_{0}$ (e.g. $(x: x s)$ in sumeuler), so that $x s_{0}$ can be included in the slice. The syntactic equivalence relation for lists is used to detect such list reconstructions.

Definition 3.4 (Syntactic Equivalence for Lists). Given some program $p$; argument $x$ of type list $\tau$, and a cons-expression $e=$ $\operatorname{cons}_{\tau} e^{\prime} e^{\prime \prime}$, we say that $e$ is syntactically equivalent to $x$, denoted $e \equiv x$, when $\exists y, \exists y s, e^{\prime}=y \wedge e^{\prime \prime} \equiv y s \wedge y \triangleleft_{p} x \wedge y s \triangleleft_{p} x$.

For example, where $x s_{0}$ is case-split in sumeuler into $\mathrm{x}$ and $\mathrm{xs}, x s_{0}$ is syntactically equivalent to a cons-expression with $x$ as the first argument and $\mathrm{xs}$ as the second argument; i.e. $x s_{0} \equiv \operatorname{cons}_{\tau} \mathrm{x} \times \mathrm{s}$.

\section{Determining Obstructiveness}

Each operation is inspected to determine whether that operation is obstructive. An obstructive operation is an operation that has either: $i$ ) any arguments whose subexpressions contain a recursive call; or ii) any arguments that are both used in the body of the fixpoint expression and whose value is changed significantly in any recursive call. All other operations are classified as unobstructive. So, for example, (euler $\mathrm{x}$ ) in sumeuler is classified as unobstructive because euler is a unary function that takes only the head of the list. Conversely, the infix addition operation in sumeuler is classified as obstructive because it takes the result of a recursive call as an argument. Obstructiveness is defined formally in Def. 4.6. The nature of significance is defined below.

\subsection{Variable Usage and Update}

From the intuitive definition of obstructiveness above, all variables in $\Gamma_{p}$ for some fixpoint expression, $e=f \operatorname{fix} \lambda\left(f, x_{1}, \ldots, x_{n}\right) \rightarrow e^{\prime}$, must be first inspected to classify operations in $e$ as (un)obstructive. Each variable $x \in \Gamma_{p}$ is classified as used, updated, or both, in $e$.

Although all the variables in $P$ are immutable, and each function application binds new values to each of its arguments, the value of some variable $x$ is considered to be (potentially) changed in $e$ when $x$ is the $i^{\text {th }}$ argument to $f$ and there exists some recursive call to $f$ in $e^{\prime}$ or any of its subexpressions. That is, the differences in the value of a specific argument between successive recursive calls are considered to be changes to the corresponding bound variables. We extend this notion with the concept of significantly changed. Intuitively, a change in value is considered significant when for 
some recursive call, $f\left(e_{1}, \ldots, e_{n}\right)$, the $i^{\text {th }}$ argument, $e_{i}$, is not: $\left.i\right)$ a variable that is reachable from $x$; ii) syntactically equivalent to $x$; nor iii) a cons-expression that prepends an expression to $x$ (or its syntactic equivalent) that contains no subexpression that is a variable reachable from $x$. Variables whose value is significantly changed in this way are classified as updated.

Definition 4.1 (Variable Update). Given a program $p$, a program environment $\Gamma_{p}$, a fixpoint expression $e=\operatorname{fix}\left(\lambda\left(f, x_{1}, \ldots, x_{n}\right) \rightarrow\right.$ $\left.e^{\prime}\right)$ in $p$, and an argument $x$, where $x$ is the $i^{\text {th }}$ argument to $f, x$ is considered to be updated in $e\left(\right.$ denoted $\bar{x} \sim_{x} e$ ) when there exists a recursive call, $f\left(e_{1}, \ldots, e_{n}\right) \ll e^{\prime}$ such that $\neg\left(e_{i} \triangleleft_{p}^{*} x\right) \wedge\left(e_{i} \not \equiv\right.$ $x) \wedge\left(e_{i} \neq \operatorname{cons}_{\tau} e_{k} e_{l} \wedge e_{l} \equiv x \wedge \nexists y \ll e_{k}, y \triangleleft_{p}^{*} x\right)$

Usage is a simpler concept: a variable is considered used when it occurs as a subexpression of a fixpoint expression. There is one exception to this: when a subexpression is the $i^{\text {th }}$ argument to a recursive call. We first define the notion of variable-usage escapement. This is to avoid the used classification of $x$, and ultimately the membership of $x$ in the slice, when $e_{i}$ is a $x$ itself or when prepending to $x$. For example, it avoids the erroneous classification of $x$ in:

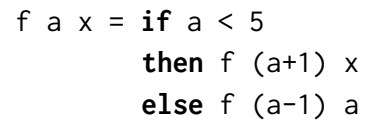

Here, in Line $3, x$ is correctly classified as being updated. In Line $2, x$ is not updated, but also should not be considered to be used, since $x$ is necessary as an argument to the recursive call in order to retain the value of $x$.

Definition 4.2 (Variable-Usage Escapement). For some fixpoint expression $e=\operatorname{fix}\left(\lambda\left(f, x_{1}, \ldots, x_{n}\right) \rightarrow e^{\prime}\right), \forall f\left(e_{1}, \ldots, e_{n}\right) \ll e^{\prime}$, and for some $i \in[1, n]$, given $x$, the $i^{\text {th }}$ argument to $f$, we substitute the special symbol $\varepsilon$ for $e_{i}$ whenever $e_{i}=x$ or $e_{i} \equiv x$. When $e_{i}=\operatorname{cons}_{\tau} e_{k} e_{l} \wedge e_{l} \equiv x \wedge \nexists y \ll e_{k}, y \triangleleft_{p}^{*} x$ holds, we substitute $\varepsilon$ for $e_{l}$. Variable-usage escapement of $e$ with respect to $x$ is denoted $e \backslash_{\varepsilon} x$.

In the above definition of $f$, for example, the $x$ in Line 2 would be substituted for $\varepsilon$.

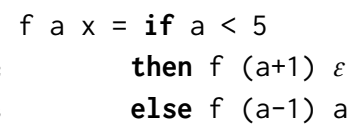

We can now define variable usage.

Definition 4.3 (Variable Usage). Given some fixpoint expression $e=\operatorname{fix}\left(\lambda\left(f, x_{1}, \ldots, x_{n}\right) \rightarrow e^{\prime}\right)$, and a variable $x$ where $x$ is the $i^{\text {th }}$ argument to $f$, a variable $y$ is considered to be used in $e$ (denoted $y \sim_{x} e$ ) when $y \triangleleft_{p}^{*} x$ and $y$ exists as a subexpression to the variableusage escaped $e$; i.e. $y \ll\left(e \backslash_{\varepsilon} x\right)$.

To illustrate this, consider the following functions.

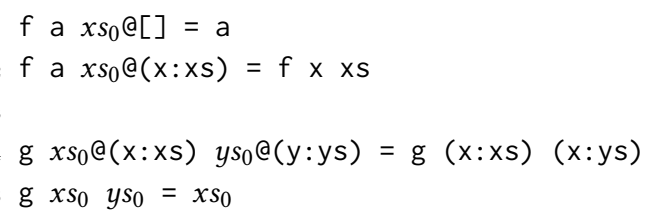

$8 \mathrm{~h} x s_{0} @(\mathrm{x}: \mathrm{xs}) \quad y s_{0} @(\mathrm{y}: \mathrm{ys})=\mathrm{h}(\mathrm{y}:(\mathrm{x}: \mathrm{xs}))(\mathrm{y}:(\mathrm{y}: \mathrm{ys}))$

9 $\mathrm{h} x s_{0} \quad y s_{0}=x s_{0}$

For $f$, the value of a is updated since $\neg\left(x \triangleleft_{p}^{*} a\right)$. However, the list argument is not updated since $\mathrm{xs}$ is reachable from $x s_{0}$; i.e. $\mathrm{xs} \triangleleft_{p}^{*} x s_{0}$. All variables, excluding $x s_{0}$, in the program environment of $f$ are considered to be used: $a$ is used in Line 1; $x$ in the first argument of the recursive call; and $x$ s in the second argument of the recursive call. For $\mathrm{g}, x s_{0}$ is not updated since $(\mathrm{x}: \mathrm{xs})$ is syntactically equivalent to $x s_{0}$; i.e. $\operatorname{cons}_{\tau} \mathrm{x} \times \mathrm{s} \equiv x s_{0}$. However, $y s_{0}$ is updated since ( $\mathrm{x}: \mathrm{ys})$ is not syntactically equivalent to $y s_{0}$. All variables apart from $y$ and $x s$ are considered to be used in $g: x s_{0}$ is used both in Line 5 and as the semantically equivalent first argument to the recursive call; $x$ and $y$ s are used in the second argument of the recursive call. Finally, for $\mathrm{h}, x s_{0}$ is not updated, since $\mathrm{y}$ (which is not reachable from $x s_{0}$ ) is prepended to $x s_{0}$. However, $y s_{0}$ is updated since $\mathrm{y}$ is prepended to $y s_{0}$ and $\mathrm{y}$ is reachable from $y s_{0} . \mathrm{y}$, $x s_{0}$, and $y s_{0}$ are all considered to be used in $\mathrm{h}: x s_{0}$ in Line 9 and the cons in the first argument of the recursive call; $y s_{0}$ in the cons in the second argument of the recursive call; $y$ in the cons of both arguments of the recursive call.

\subsection{Slicing Algorithm}

Intuitively, a slice $\Sigma^{e \mid x}$ of an expression $e$ with criterion $x$ is a set of variables that indicates whether a variable $y$ is used in $e$ and whether $x$ is updated in $e$, denoted by the annotation $\bar{x}$. A slice can be used to categorise variables, and ultimately to determine the obstructiveness of operations.

Definition 4.4 (Slice). Given some program $p$, the program environment $\Gamma_{p}$, a fixpoint expression $e=\operatorname{fix} \lambda\left(f, x_{1}, \ldots, x_{n}\right) \rightarrow e^{\prime}$, and a variable $x$, where $x$ is the $i^{\text {th }}$ argument to $f$, we say that the slice of $e$ with criterion $x$, denoted $\Sigma^{e \mid x}$, is the set of variables such that $\forall y \in \Sigma^{e \mid x},\left(y \sim_{x} e\right) \vee\left(y=\bar{x} \wedge \bar{x} \sim_{x} e\right)$.

To illustrate the slicing relation, recall the definition of sumeuler,

sumeuler $x s_{0} @[]=0$

sumeuler $x s_{0} @(\mathrm{x}: \mathrm{xs})=$ euler $\mathrm{x}+($ sumeuler $\mathrm{xs})$

Considering each variable in sumeuler: both $\mathrm{x} \in \Sigma^{\text {sumeuler }} \mid x s_{0}$ and $\mathrm{xs} \in \Sigma^{\text {sumeuler } \mid x s_{0}}$ hold since both $\mathrm{x}$ and $\mathrm{xs}$ are reachable from $x s_{0}$ (i.e. $\mathrm{x} \triangleleft_{p}^{*} x s_{0}$ and $\mathrm{xs} \triangleleft_{p}^{*} x s_{0}$ ) and both $\mathrm{x}$ and $\mathrm{xs}$ are used in Line 2; conversely, neither $x s_{0} \in \Sigma^{\text {sumeuler } \mid x}$ nor $x \bar{s}_{0} \in \Sigma^{\text {sumeuler } \mid x s_{0}}$ hold, since $x s_{0}$ is not considered used, and $x s_{0}$ is not considered updated, in sumeuler. For the definition of sum:

sum $x \quad y s_{0} @[]=x$

sum $x y s_{0} @(y: y s)=\operatorname{sum}(x+y)$ ys

$x \in \Sigma^{\text {sum } \mid x}$ holds since $x$ is used in both Lines 1 and $2 . \bar{x} \in \Sigma^{\text {sum } \mid x}$ also holds since $x$ is updated in the recursive call in Line 2 by the $(x+y)$ operation. As with sumeuler, both $y \in \Sigma^{\text {sum } \mid y s_{0}}$ and ys $\in \Sigma^{\text {sum } \mid y s_{0}}$ hold since both y and ys are considered to be used in Line 2. Conversely, neither $y s_{0} \in \Sigma^{\text {sum }} \mid y s_{0}$ nor $y \bar{s}_{0}$ hold since $y s_{0}$ is not considered to be used or updated in sum. The slices of sumeuler and sum can be presented:

$\begin{array}{ll}\sum^{\text {sumeuler } \mid x s_{0}} & =\{\mathrm{x}, \mathrm{xs}\} \\ \Sigma^{\text {sum } \mid \mathrm{x}} & =\{\mathrm{x}, \overline{\mathrm{x}}\} \\ \Sigma^{\text {sum } \mid y s_{0}} & =\{\mathrm{y}, \mathrm{ys}\}\end{array}$




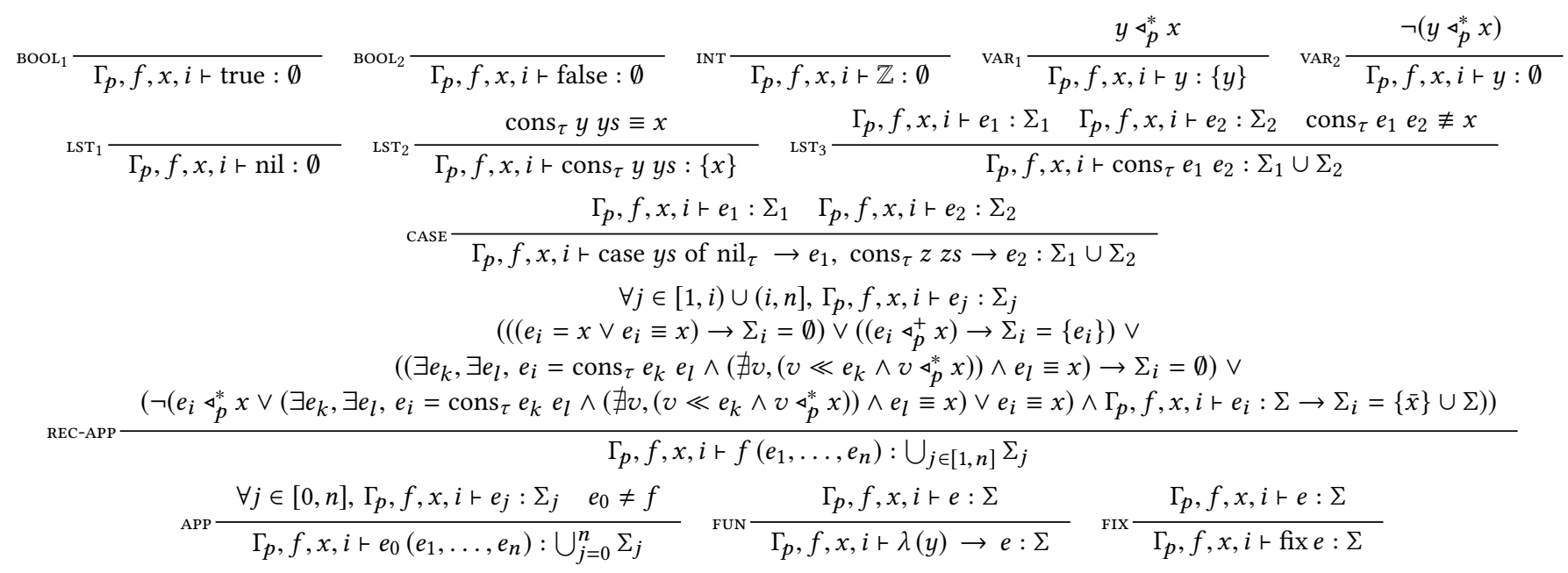

Figure 4. Inference rules to calculate the slice $\Sigma^{e \mid x}$ for an expression $e$ with criterion $x$.

The slice $\Sigma^{e \mid x}$ is calculated using the inference rules from Fig. 4. For clarity of notation, we will write $\Sigma^{e \mid x}$ as $\Sigma$ since neither $e$ nor $x$ can be changed within a slicing operation; showing that $\Sigma=\Sigma^{e \mid x}$ remains future work. Judgements are in the form:

$$
\Gamma_{p}, f, x, i \vdash e: \Sigma
$$

where $\Gamma_{p}$ is the environment for some program $p ; f$ is the name of the fixpoint function being sliced; $x$ is the slicing criterion that is declared as the $i^{\text {th }}$ argument of $f$; $e$ is the expression in $p$ that is being sliced; and $\Sigma$ is the resulting slice. For literal expressions and variables that are not reachable from $x, e$ produces an empty slice, represented by the rules $\mathrm{BOOL}_{1}, \mathrm{BOOL}_{2}, \mathrm{INT}_{2} \mathrm{VAR}_{2}$, and LST 1 . A variable that is reachable from $x$, as a usage of $x$, produces the slice containing that variable $\left(\mathrm{VAR}_{1}\right)$. Cons-expressions that are syntactically equivalent to $x$ produce the slice containing $x$ itself $\left(\mathrm{LST}_{2}\right)$. All other expressions that are not recursive calls produce the union of the slices of their subexpressions, as stated in the rules $\mathrm{LST}_{3}$, CASE, APP, FUN, and FIX. Finally, REC-APP determines whether $x$ is updated in a recursive call, producing the appropriate slice. REC-APP has two main premises that: $i$ ) slice all subexpressions that are passed to $f$, aside from the $i^{\text {th }}$ argument; and $i$ ) determines whether $x$ is updated. The second premise is a disjunction of four implications: $i$ ) when $e_{i}$ is $x$ itself, or is syntactically equivalent to $x$; ii) when $e_{i}$ is a variable that is defined via case-discrimination on $x$; iii) when $x$ is prepended by some expression $e_{k}$ that does not contain a subexpression that is a variable reachable from $x$; and otherwise $i v$ ) when $x$ is considered updated. In the first case, the slice, $\Sigma_{i}$, for the $i^{\text {th }}$ argument to $f, e_{i}$, is the empty set since the argument preserves the value of $x$ for the next recursive call. In the second case, $\Sigma_{i}$ is the singleton set containing $e_{i}$ itself since the value of $x$ is changed, and a case-derived variable is used, but $x$ is not considered to be updated. In the third case, $\Sigma_{i}$ is the slice of the subexpression that is prepended to $x$. Finally, in the fourth case, $\Sigma_{i}$ is the slice of $e_{i}$ and $x$ is considered to be updated.

We conjecture that the slicing algorithm in Fig. 4 is sound with respect to the slicing definition, Def. 4.4, such that our algorithm produces a slice whose members are only those variables that are considered to be used or updated in $e$ with respect to $x$. Similarly, we conjecture that slices are unique for each $e$ and $x$. We defer the proofs of soundness and uniqueness to future work.

\subsection{Classifying Variables}

Variables can be classified as: global, clean, or tainted. Global variables are those that are in scope in $e$, but which are declared and bound outside of $e$. They may be used, but cannot be updated during the evaluation of $e$; they are therefore treated as literals. Variables that are classified as either clean or tainted are ones that are either defined in the fixpoint function (i.e. $x_{1}, \ldots, x_{n}$ ), or in casesubexpressions of $e^{\prime}$, where whenever $\forall v, w \in \Gamma_{p}$ such that $v \triangleleft_{p}^{+} w$, it follows that $v$ has the same classification as $w$.

Definition 4.5 (Variable Taint). Given a program $p$, program environment $\Gamma_{p}$, a fixpoint expression $e=\operatorname{fix}\left(\lambda\left(f, x_{1}, \ldots, x_{n}\right) \rightarrow e^{\prime}\right)$ in $p$, a variable $x$, and a slice $\Sigma^{e \mid x}$ of $e$ with criterion $x$, then $x$ is classified as tainted when $\bar{x} \in \Sigma^{e \mid x} \wedge x \in \Sigma^{e \mid x}$. The variable is classified as clean, otherwise.

\subsection{Classifying Operations}

Once all the arguments of $e$ are classified as global, clean, or tainted, we can proceed to classify those non-recursive application subexpressions of $e$, i.e. the operations in $e$. Those operations that take one or more variables that are classified as tainted are themselves classified as obstructive. All other operations are classified as unobstructive.

Definition 4.6 (Operation Obstructiveness). Given some program $p$, some program environment $\Gamma_{p}$, and some fixpoint expression $e=\operatorname{fix}\left(\lambda\left(f, x_{1}, \ldots, x_{n}\right) \rightarrow e^{\prime}\right)$ in $p$, an operation $g\left(e_{1}, \ldots, e_{m}\right) \ll$ $e^{\prime}$, when $g \neq f$, is classified as obstructive when $\exists i \in[1, m]$ such that $f\left(\vec{e}_{f}\right) \ll e_{i} \vee \exists y, y \ll e_{i}$ where $y$ is classified as tainted. The operation is classified as unobstructive otherwise.

\section{Examples}

In this section we demonstrate our approach on 12 examples, including sumeuler, matrix multiplication, n-queens, and the SmithWaterman algorithm. We give parallel speedup results for sumeuler, matrix multiplication, and $n$-queens. Our source code is available 


\begin{tabular}{|c|c|c|c|c|c|c|c|c|c|c|}
\hline \multirow{2}{*}{ Example } & \multirow{2}{*}{ RFs } & \multirow{2}{*}{ Ops } & \multirow{2}{*}{$\mathrm{RCs}$} & \multirow{2}{*}{ Args } & \multicolumn{2}{|c|}{ Unobstructive Ops } & \multicolumn{2}{|c|}{ Obstructive Ops } & \multirow{2}{*}{$\begin{array}{c}\text { Time } \\
(\mu s)\end{array}$} & \multirow{2}{*}{$\begin{array}{c}\sigma \\
(\mu s)\end{array}$} \\
\hline & & & & & Actual & Found & Actual & Found & & \\
\hline Data.List & 18 & 22 & 20 & 31 & 5 & 5 & 17 & 17 & 6797.22 & 409.14 \\
\hline elem & 1 & 2 & 1 & 2 & 1 & 1 & 1 & 1 & 176.28 & 26.51 \\
\hline list-ackermann & 1 & 0 & 3 & 2 & 0 & 0 & 0 & 0 & 196.46 & 20.65 \\
\hline gcd & 1 & 3 & 1 & 2 & 0 & 0 & 3 & 3 & 178.46 & 48.93 \\
\hline hanoi & 1 & 5 & 1 & 1 & 0 & 0 & 5 & 5 & 156.16 & 39.50 \\
\hline k-means & 4 & 15 & 5 & 14 & 4 & 4 & 11 & 11 & 646.30 & 60.90 \\
\hline quicksort & 3 & 6 & 5 & 5 & 2 & 2 & 4 & 4 & 350.94 & 17.98 \\
\hline swaterman & 2 & 10 & 2 & 10 & 0 & 0 & 10 & 10 & 420.92 & 18.44 \\
\hline sumeuler & 14 & 47 & 14 & 25 & 16 & 16 & 31 & 31 & 2171.36 & 63.95 \\
\hline matmult & 8 & 23 & 7 & 16 & 6 & 6 & 17 & 17 & 1195.62 & 63.23 \\
\hline queens & 2 & 11 & 2 & 6 & 2 & 2 & 9 & 9 & 487.04 & 24.48 \\
\hline sudoku & 5 & 9 & 5 & 8 & 3 & 3 & 6 & 6 & 519.66 & 38.68 \\
\hline
\end{tabular}

Figure 5. Examples run through prototype implementation. Times are an average of 50 runs.

at https://adb23.host.cs.st-andrews.ac.uk/fhpc17-parallel.zip. Our results show that our approach can discover map operations that lead to real performance improvements. Speedups are an average of five runs on corryvreckan, a $2.6 \mathrm{GHz}$ Intel Xeon E5-2690 v4 machine with 28 physical cores and 256GB of RAM. This machine allows turbo boost up to $3.6 \mathrm{GHz}$, and supports automatic dynamic frequency scaling between $1.2-3.6 \mathrm{GHz}$. Speedups use reported mutator time, i.e. the amount of time spent solely on executing the program. This gives an indication of real parallel performance. Where we use corryvreckan for parallel speedup results, we use a separate, standard desktop machine, neptune, to test our prototype implementation. neptune is a $2.7 \mathrm{GHz}$ Intel Core 15 machine with 8GB of RAM, running Mac OS X 10.11.6 and Erlang 19.2.3. The parallel speedup results, including speedup graphs in Figs. 1, 7, and 8 are reiterated from [4], where we expand upon the results reported here. sumeuler, queens, and matmult have all been compiled using GHC 7.6.3 on Scientific Linux version 3.10.0. We compiled the examples using the flags: -feager-blackholing, -threaded, -rtsopts, and -0 . We found these gave the best general parallel performance.

We have implemented our inference system in Fig. 4 in Erlang, providing a parser for our expression language and a classifier for operations. For a given program $p$, we first produce the environment. Then for each fix expression $e=\operatorname{fix} \lambda(f, \vec{x}) \rightarrow e^{\prime}$ in $p$, we slice $e$ with respect to each argument $x \in \vec{x}$. Each $x$ is classified as either clean or tainted. This is then used to classify each operation in $e$ either obstructive or unobstructive. Our implementation can be found at http://adb23.host.cs.st-andrews.ac.uk/fhpc17-artefact.zip.

The NoFib benchmarks have all been translated from Haskell; if-expressions have been translated as application expressions, and so count as operations. Our other examples, including the 18 functions from the Haskell Prelude Data.List library, may not all benefit from parallelisation, but serve as a demonstration of our approach. The 18 examples chosen from Data.List are: and, or, append, foldl, foldr, init, intersperse, last, length, map, maximum, replicate, reverse, scan, heads, subsequences, tails and transpose. These are a representative subset of the functions in the library; the remaining functions are similar to these. All our (translated) example code can be found at https: //adb23.host.cs.st-andrews.ac.uk/fhpc17-examples.zip.

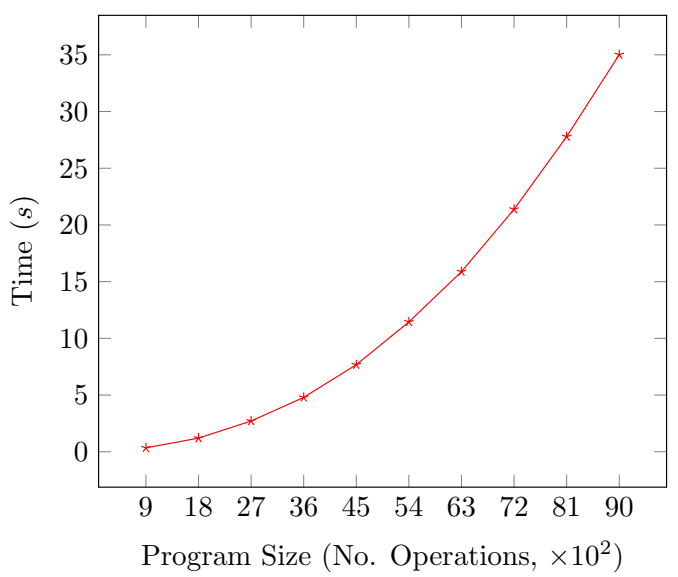

Figure 6. Prototype execution times for a program with varying sizes of input. Our implementation is quadratic.

We give an overview of our results in Fig. 5, where gcd refers to the greatest common denominator function, hanoi refers to the Towers of Hanoi puzzle, and swaterman refers to the SmithWaterman algorithm. The list-ackermann example is a reimplementation of the standard Ackermann function that traverses lists.

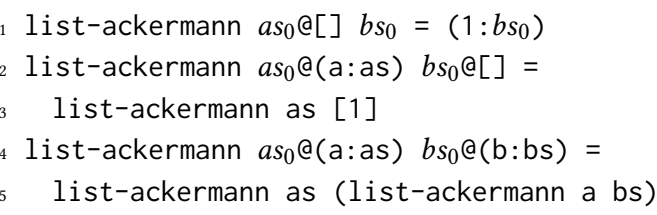

Our results show that all operations are correctly classified. For each example, we give: the number of recursive functions that it contains (RFs), the total number of operations (Ops), the total number of recursive calls (RCs), the total number of arguments to all recursive functions (Args), the expected number of obstructive and unobstructive operations (Actual), those that are found by our prototype (Found), the average execution time for our prototype of 50 runs on neptune for that example (Time), and standard deviation $(\sigma)$ of those times. 
As a synthetic benchmark, we have also applied our prototype to programs with varying input sizes, measured in the number of operations, by duplicating the translated SumEuler module $m$ times. As the translated SumEuler has 9 operations, the total number of operations is $n=m \times 9$. Fig. 6 shows the average time taken of five runs, in seconds, by our prototype on neptune. Our classifier takes a minimum of $0.35 \mathrm{~s}$ for $n=900$, with a standard deviation of $0.03 s$, and a maximum of $35.02 s$ for $n=9000$, with a standard deviation of $0.58 \mathrm{~s}$. Our prototype implementation runs in quadratic time with respect to $n$. The total time taken to classify each of our examples on neptune are all low, as shown in Fig. 5. The longest our prototype takes to classify one of the examples in Fig. 5 is $6.80 \mathrm{~ms}$.

\subsection{Sumeuler}

Recall that sumeuler applies Euler's totient function to a list of integers and sums the result.

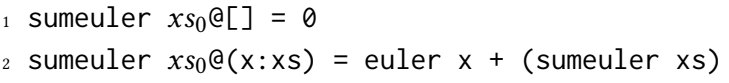

We first slice sumeuler for its only argument, $x s_{0}$, $\Sigma^{\text {sumeuler }} \mid x s_{0}=$ $\{x, x s\}$. As before, $x s_{0}$ is classified as clean since both case-split variables of $x s_{0}$ ( $\mathrm{x}$ and $\mathrm{xs}$ ) are used in the body of sumeuler, and $x s_{0}$ itself is not updated. Two operations exist as subexpressions to sumeuler: (euler $\mathrm{x}$ ) and the application of $(+)$ in Line 2 . (euler $\mathrm{x}$ ) takes a single clean argument, i.e. $\mathrm{x}$, and is classified as unobstructive. Conversely, $(+)$ takes two arguments, where the second argument comprises a recursive call, and is classified as obstructive. (euler $\mathrm{x}$ ) can be lifted into a map operation, and sumeuler is rewritten to introduce chunking and parallelism using the Strategies library. We are also able to take advantage of the associativity of $(+)$, summing each chunk before summing the result of all chunks.

In [4] we executed sumeuler for $n=1,000$ and between 5,000 and 50,000 at intervals of 5,000, with a chunk size of 500. Fig. 1 gives the speedups for sumeuler using mutator time. We achieve maximum speedups of 30.50 for $n=50000$ on 48 virtual cores. Sequentially, when $n=50000$ sumeuler has an average runtime of $154.33 \mathrm{~s}(\sigma=0.25 \mathrm{~s})$. We achieve good speedups in all cases. Moreover, sumeuler demonstrates good scalability as $\mathrm{n}$ increases. Fig. 1 shows that for varying $\mathrm{n}$, speedups reach a limit, likely due to lack of work.

Our translation of the NoFib example code comprises 14 recursive functions over three Haskell modules. Within those 14 recursive functions, there are a total of 47 operations, 16 of which are classified unobstructive and 31 of which are classified as $o b-$ structive. As with all the NoFib benchmarks, the high number of recursive functions are representative of the original duplication in the underlying Haskell modules.

\subsection{Matrix Multiplication}

The NoFib benchmark uses the following implementation of matrix multiplication.

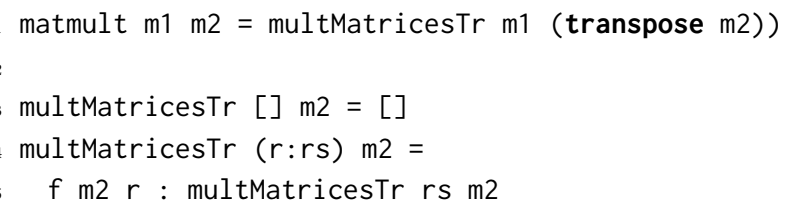

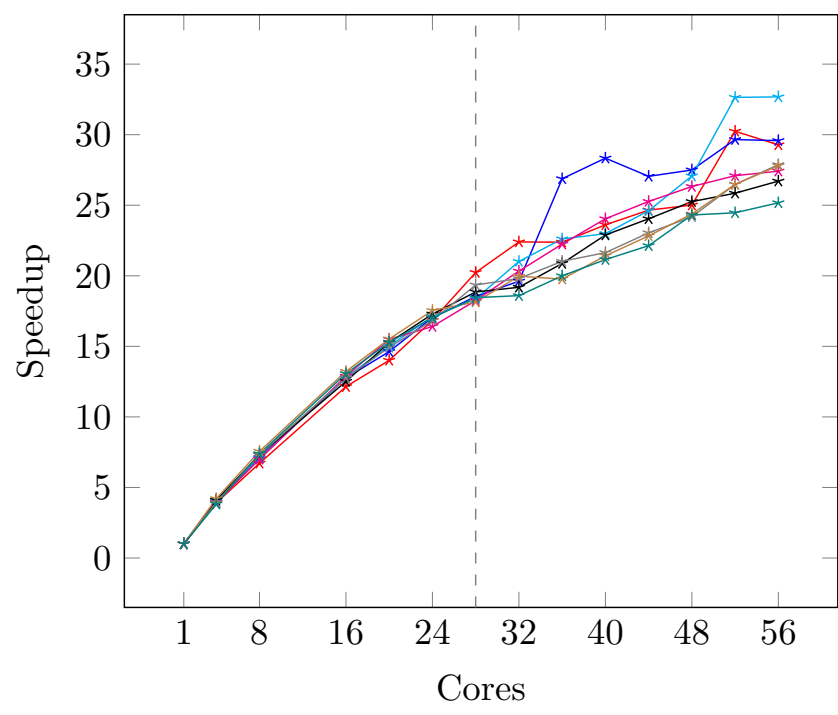

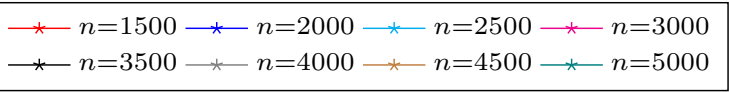

Figure 7. matmult, speedups on corryvreckan using reported mutator (MUT) time, dashed line shows extent of physical cores.

7 f $c s_{0} @[]$ row $=[]$
8 f $c s_{0} @(c: c s)$ row $=$ prodEscalar2 row $c: f$ cs row

Here, transpose transposes a matrix and prodEscalar 2 calculates the dot product of two lists. Since matmult is not a fix expression, we slice for all the arguments to multMatricesTr.

$$
\Sigma^{\text {multMatricesTr } \mid r s_{0}}=\{r, r s\} \quad \sum^{\text {multMatricesTr } \mid \mathrm{m} 2}=\{\mathrm{mt}\}
$$

As with our other examples, both of the arguments are used but not updated and are therefore classified as clean. The sole operation, ( $f \mathrm{~m} 2 \mathrm{r}$ ), is classified as unobstructive. Slicing for all arguments of $f$,

$$
\Sigma^{f \mid c s_{0}}=\{c, c s\} \quad \Sigma^{f \mid r o w}=\{\text { row }\}
$$

we can see the same pattern: all the arguments are classified clean, and the sole operation, (prodEscalar2 row c), is classified as unobstructive. Both multMatricesTr, and $f$ can be rewritten as map operations. Parallelism can be introduced in matmult by applying a custom strategy to the call to multMatricesTr that chunks matrices into blocks, rather than along rows or columns. In [4] we executed matmult for $n$ between 1000 and 5000 at intervals of 500, with the chunk size set to 20 . Fig. 7 gives speedups for matmult using mutator time, achieving maximum a speedup of 32.93 for $n=2500$ on 52 hyper-threaded cores. Sequentially, when $n=2500$ matmult takes an average of 226.58 s, with a standard deviation of 7.49s. matmult scales well for both $\mathrm{n}$ and number of cores. On hyperthreaded cores we observe some erratic behaviour with curious superlinear speedups. This is possibly due to caching effects.

Interestingly, the definition of $f$ might be unfolded (in the transformational sense) in multMatricesTr; e.g.

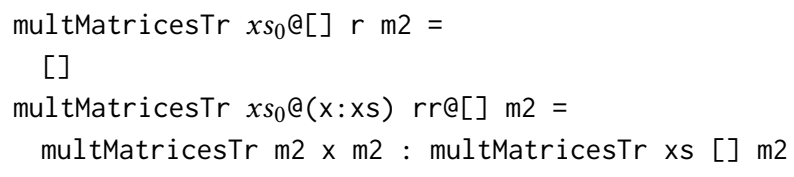


multMatricesTr $x s_{0} @(\mathrm{x}: \mathrm{xs}) \mathrm{rr} \mathrm{m} 2=$

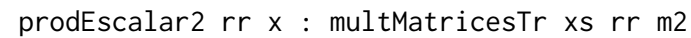

Here, multMatricesTr is now a fix-expression, case-splitting on its first argument, $x s_{0}$. Slicing for its arguments, gives

$$
\begin{aligned}
\sum^{\text {multMatricesTr } \mid x s_{0}} & =\left\{\mathrm{x}, \mathrm{xs}, x \bar{s}_{0}\right\} \\
\Sigma^{\text {multMatricesTr } \mid r r} & =\{\mathrm{rr}, \mathrm{r} \bar{r}\} \\
\Sigma^{\text {multMatricesTr } \mid \mathrm{m} 2} & =\{\mathrm{m} 2\}
\end{aligned}
$$

While $x s_{0}$ is considered to be updated, due to $\mathrm{m} 2$ being passed as the first argument in the recursive call in line Line 4, both $x s_{0}$ and $\mathrm{m} 2$ are considered to be clean. Conversely, $r r$ is considered to be tainted. (prodEscalar2 $r r \times$ ), now the sole operation, is therefore classified obstructive, meaning no map operations can be introduced.

This is a correct result since mul tMatrices $T r$ traverses two lists, where both the lists are passed as the first argument. The standard definition of map, for example, does not allow this behaviour, and any attempt at introducing a map operation would result in a function that is not functionally equivalent to multMatricesTr. This limitation arises again in queens with fused mutually recursive functions. A map is only discoverable when the inspected function recurses over one or more data structures and where at least one argument is structurally smaller for each recursive call.

Our translation of the NoFib benchmark code comprises 8 recursive functions over 2 Haskell modules. Within those 8 recursive functions there are 23 operations, of which 6 are classified as unobstructive and 17 are classified as obstructive.

\section{$5.3 \quad \mathrm{~N}$-Queens}

We use the following implementation of the queens problem,

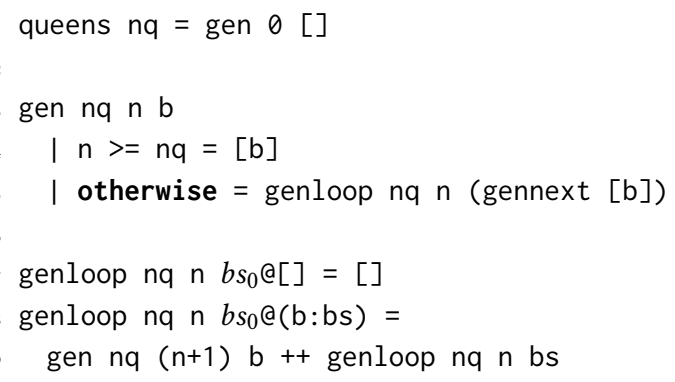

where $n q$ is the number of queens and gennext calculates the position of a queen on a board. We slice for all arguments of genloop.

$$
\Sigma^{\text {queens } \mid \mathrm{nq}}=\{\mathrm{nq}\} \quad \Sigma^{\text {queens } \mid \mathrm{n}}=\{\mathrm{n}\} \quad \Sigma^{\text {queens }} \mid b s_{0}=\{\mathrm{b}, \mathrm{bs}\}
$$

All three arguments, nq, $\mathrm{n}$, and $b s_{0}$, are classified as clean since both nq and $\mathrm{n}$ are used but not updated; and both $\mathrm{b}$ and bs are used but $b s_{0}$ is neither used nor updated. genloop has three subexpressions: $i)(n+1)$ in the call to gen; ii) (gen nq $(n+1)$ b) in the first argument to $(++)$; and iii) the top-level $(++)$. Both $(n+1)$ and (gen $\mathrm{nq}(\mathrm{n}+1)$ b) are classified as unobstructive since only clean variables occur the expressions passed to $(+)$ and gen respectively. We additionally note that (gen $n q(n+1)$ b) contains the unobstructive operation $(n+1)$ as a subexpression. Hypothetically, if $(n+1)$ had been classified as obstructive, then (gen nq $(n+1)$ b) must also be classified as obstructive. While no tainted variables occur in the arguments to the append operation, its second argument is a recursive call, which results in an obstructive classification. Although it is possible to lift either unobstructive operations

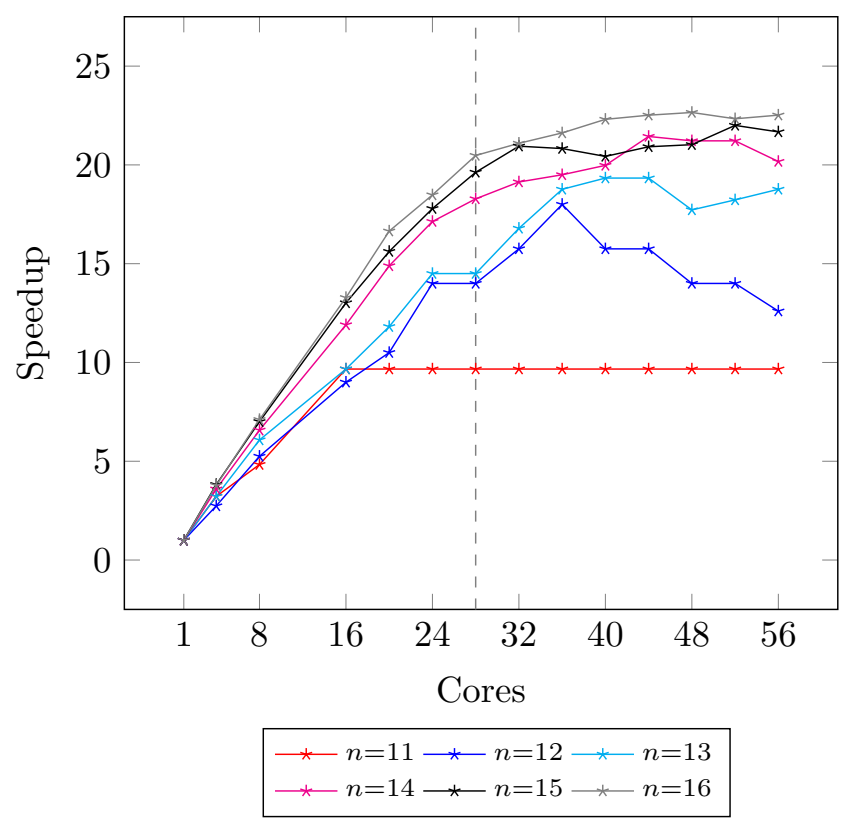

Figure 8. queens, speedups on corryvreckan using reported mutator (MUT) time, dashed line shows extent of physical cores.

into a map operation, we choose to lift the operation which does not occur as a subexpression of any unobstructive operation, i.e. (gen nq $(n+1)$ b), to maximise the amount of work done in parallel.

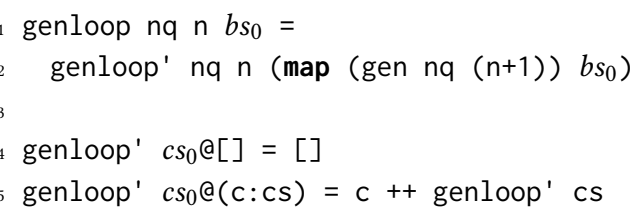

Similar to $f$ and multMatricesTr in matmult, we observe that gen and genloop could be merged into a single definition.

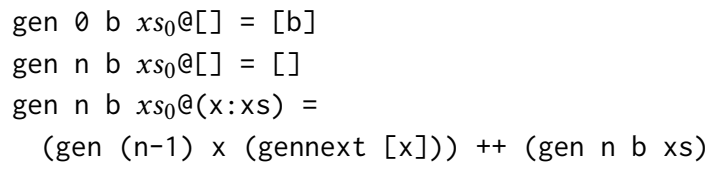

This definition eliminates the mutual recursion of gen and genloop, where $x s_{0}$ traverses two different lists and $\mathrm{n}$ acts as an additional bound on the recursion. As before, our technique will (correctly) classify all operations as obstructive. In this example, it is the recursive call in the first argument to $(++)$ that introduces an update to all variables.

Our translation of the queens NoFib implementation comprises two recursive functions with 11 operations, and 2 recursive calls. Despite the number of operations in queens, there are only two unobstructive operations in the translation. The majority of operations are found in the definition of safe.

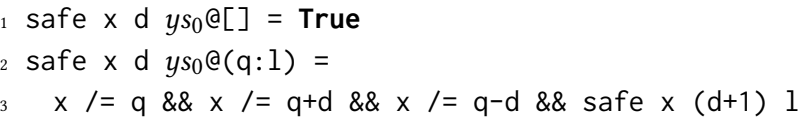


Slicing safe for all arguments,

$$
\Sigma^{\text {safe } \mid \mathrm{x}}=\{\mathrm{x}\} \quad \Sigma^{\mathrm{safe} \mid \mathrm{d}}=\{\mathrm{d}, \overline{\mathrm{d}}\} \quad \Sigma^{\text {safe } \mid y s_{0}}=\{\mathrm{q}, 1\}
$$

we classify $x$ and $y s_{0}$ as clean and $\mathrm{d}$ as tainted. Here, the tainted classification of $d$ results in obstructive classifications of all but one infix inequality operations. Hypothetically, if $d$ had been classified as clean, we observe that how the infix (\&\&) operations are compiled can affect the classifications of operations in safe. Parsing (\&\&) as either left- or right-associative can produce different numbers of obstructive operations. Here, parsing (\&\&) as left-associative minimises the number of obstructive operations; i.e. when,

$1(x /=q \& \& x /=q+d) \& \&(x /=q-d \& \&$ safe $x(d+1) 1)$

only the topmost (\&\&) and its second argument are classified as $o b$ structive. Conversely, parsing (\&\&) as right-associative maximises the number of obstructive operations; i.e. when,

$1 \times /=q \& \&(x /=q+d \& \&(x /=q-d \& \&$ safe $x(d+1) 1))$

all (\&\&) operations are classified as obstructive.

The queens example is parallelised by applying a strategy from the Strategies library to the map. To ensure that parallelism is worthwhile, the specific strategy used (parallel or sequential) is passed in as an argument. A threshold argument is then added to gen to control the depth to which the map operation is performed in parallel. We executed queens for $n$ ranging from 11 to 16 , with a threshold depth of 2. Fig. 8 show speedups for queens in terms of mutator time. We achieve maximum speedups of 22.65 for $n=16$ on 48 hyperthreaded cores. Sequentially, when $n=16$, queens takes an average of 717.66s $(\sigma=0.25 \mathrm{~s})$. When $n=11$, speedups plateau before the physical cores are exhausted, likely due to a lack of work as in sumeuler. When $14 \leq n \leq 16$, queens scales well until 28 cores; when hyperthreading is enabled, we see some further improvement in speedup, albeit at reduced rate when compared with physical cores. For $n=13$ and $n=14$, we see intermediate scalability continue between 28 and 40 hyperthreaded cores. This may be due to the lack of chunking of $b s_{0}$, resulting in inefficient use of cores up to 28 cores, with hyperthreading enabling saturation of the hardware. For $n=13$ and $n=14$, we observe a similar effect to $n=11$ but at a higher level due to increased availability of work.

\subsection{Smith-Waterman}

The Smith-Waterman algorithm compares the similarity of two strings. The algorithm was originally designed for the comparison of nucleotides [36]. It has two stages: populating a matrix, and backtracking over the matrix to find the shortest 'distance' between the two strings. The code below concerns part of the matrix population stage. It traverses the matrix m, updating each cell with the result of $h$, where $h$ (implementation omitted) calculates the maximum similarity score between the two strings $a$ and $b$ for the current row $r$ and column $c$ and updates the cell with that score.

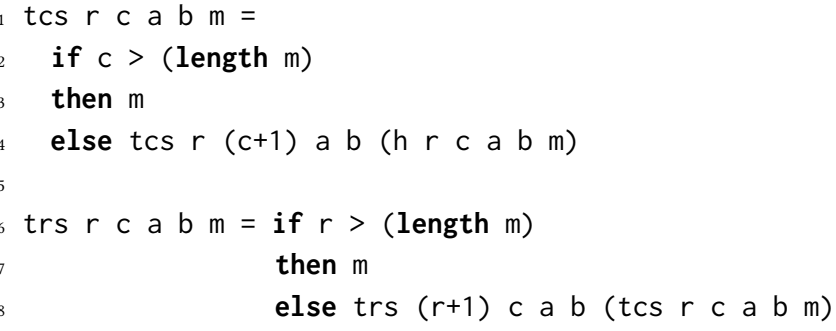

10 traverse $\mathrm{m} \mathrm{a} \mathrm{b}=\operatorname{trs} 11 \mathrm{a} \mathrm{b} \mathrm{m}$

As before, we calculate a slice for each argument to each function, classifying those arguments using the slice.

tcs:

$$
\begin{aligned}
& \Sigma^{\mathrm{tcs} \mid \mathrm{r}}=\{r\} \quad \text { (clean) } \\
& \Sigma^{\mathrm{tcs} \mid \mathrm{c}}=\{\mathrm{c}, \overline{\mathrm{c}}\} \quad \text { (tainted) } \\
& \Sigma^{\mathrm{tcs} \mid \mathrm{a}}=\{\mathrm{a}\} \quad(\text { clean }) \\
& \Sigma^{\mathrm{tcs} \mid \mathrm{b}}=\{\mathrm{b}\} \quad \text { (clean) } \\
& \Sigma^{\mathrm{tcs} \mid \mathrm{m}}=\{\mathrm{m}, \overline{\mathrm{m}}\} \quad \text { (tainted) } \\
& \Sigma^{\operatorname{trs} \mid r}=\{r, \bar{r}\} \quad \text { (tainted) } \\
& \Sigma^{\text {trs } \mid \mathrm{c}}=\{\mathrm{c}\} \quad(\text { clean }) \\
& \Sigma^{\text {trs } \mid \mathrm{a}}=\{\mathrm{a}\} \quad(\text { clean }) \\
& \Sigma^{\text {trs } \mid \mathrm{b}}=\{\mathrm{b}\} \quad \text { (clean) } \\
& \Sigma^{\text {trs } \mid \mathrm{m}}=\{\mathrm{m}, \overline{\mathrm{m}}\} \quad \text { (tainted) }
\end{aligned}
$$

trs:

Using these classifications, we can classify the operations within the recursive functions.

tcs: As both $\mathrm{c}$ and $\mathrm{m}$ are classified tainted, all operations in tcs (i.e. length, and the less-than and addition operators) are classified to be obstructive.

trs: Analogous to tcs, and as both $r$ and $\mathrm{m}$ are classified tainted, all operations in trs are classified to be obstructive.

No refactoring can be applied to the Smith-Waterman implementation. However, the Smith-Waterman algorithm is an example of wavefront parallelism whereby cells of the matrix are divided into groups which can be calculated in parallel. As our classification looks only for independence across an entire data structure, at present this goes undetected, but is part of future work.

\section{Related Work}

Program slicing was first introduced by Mark Weiser in 1981 [38] for imperative, procedureless programs. Slicing has since seen numerous extensions and adaptations for a range of fields, e.g. testing and debugging [34]. Elsewhere, intra-function slicing has also been used to introduce patterns for concurrency in Erlang code [27]. We ourselves have used slicing to transform the datatypes of Erlang functions in a slice, in order to take advantage of properties, e.g. to reduce copying overheads when sending between processes [2]. Similar to our own approach, Ahn and Han [1], use program slicing to categorise function parameters for detecting loci of parallelism. Their approach is limited to first-order languages, whereas our approach is also suitable for higher-order languages. It is designed to only inspect recursive functions that can be transformed into a form where each constructor has exactly one clause, and where functions have only a single argument. In contrast, our approach inspects functions in any form to maximise the number of components that are available for parallelisation. Even if the introduced maps are eventually deemed not worth parallelising, the more components available for inspection, the more configurations of parallelism that can be considered. Moreover, map operations, and recursion schemes in general, can encapsulate properties, e.g. independence of operation or type of output, that can be used for other purposes; e.g. termination checking. Affine types [33] and single-threadedness [20] both have their foundations in linear logic, wherein objects must be used exactly once. Affine types weaken this constraint by allowing objects to be used at most once. There 
is some superficial similarity with our technique, such that if an input can be used at most once within a recursive function then it follows that all operations that use that input can be performed independently of successive recursive calls. Single-threadedness shares a similar goal, but places greater emphasis on exposing mutation to state without sacrificing referential transparency. Again, this is superficially similar to our approach in the exposition of how an input changes between two points. However both of these approaches require a specific language or system to function. In contrast, our approach is fully generic. We additionally note that our system allows a weaker sense of usage (in that an input can be used more than once) whilst still enabling us to reason about the independence of operations.

Structured parallelism approaches, such as those used here, have been shown to be an effective means to introduce and manipulate parallelism [9, 15, 16, 25, 29]. Pattern discovery for parallelism has primarily focussed on approaches for imperative programs by analysing dependencies and machine learning techniques $[1,6,14$, 21]. The polyhedral model, in particular, uses control and data dependencies to reorder statements in code. One limitation of the polyhedral model is that it requires the AST to be translated into a specific linear-algebraic form, and not all statements can be translated to this form [5]. Alternative automatic approaches derive parallel implementations from small programs or specifications, commonly exploiting the Bird Meertens formalism [13, 17, 19, 24, 26]. These approaches use algorithmic structures, e.g. list homomorphisms [18] and hylomorphisms [9], which are amenable to divide-and-conquer skeletons. These approaches require operations to be translated into specific types which can be easily parallelised [13,26]. Similar to our own approach, Hu, Takeichi, and Iwasaki's Diffusion [24] uses program transformation techniques to rewrite explicitly recursive functions into compositions of recursion schemes. Instead of using slicing to determine operations that can be lifted into scheme fixpoint functions, Hu et al. use the the Bird Meertens formalisms to systematically reason about the behaviour of the function. This approach has been shown to extend to types other than lists, and schemes other than maps. While our approach can in principle be extended to other schemes, the slicing approach requires that the schemes are encoded in terms of variable usage and update. The diffusion approach is however, reliant on external normalisation processes that must be proven sound, and which can be a limiting factor. Another limiting factor can be found in the applicability of the language to which diffusion is applied: since it relies upon the Bird Meertens formalism, it may be difficult to extend the approach, including the necessary normalisation processes, to languages such as $\mathrm{C}++$. Our approach may prove simpler to extend to such languages, providing that state is explicit and variable usage and update are defined. One exception to the program calculation approaches is Cook's approach, which uses higher-order unification to discover and introduce common sequential patterns [12]. This approach is, however, limited in that it uses a proof assistant as an intermediate representation, and further requires that functions have a structure that mirrors the structure of the pattern to be discovered. More generally, program calculation and unification approaches are limited by the information that they are able to infer from code. Program shaping approaches [3] that include pattern discovery techniques can potentially find more patterns by refactoring code prior to pattern discovery. Developed from early work on a fold/unfold transformation system by Burstall and Darlington [8], refactoring techniques change the structure of a program while preserving functional equivalence [7]. Refactoring tools automate this transformation process and exist for many languages [7, 28] and IDEs [23]. Recent work on refactoring for parallelism has demonstrated that a refactoring approach can aid in the introduction of skeletons, but also that refactorings can enable the introduction of parallelism [2, 3, 6, 7]. While these approaches can take advantage of programmer knowledge, e.g. associativity of operations, they also require the programmer to know when and where to apply the refactorings. The approach presented here places no restriction or requirement on the structure of recursive functions, and can be included as part of a program shaping workflow to guide the application of refactorings. Moreover, our approach also allows the programmer to apply transformations that would be unreachable in fully automatic approaches, such as those described above.

\section{Conclusions and Future Work}

In this paper, we have investigated the use of program slicing to discover mappable, and by extension potentially parallelisable, computations in recursive functions. We have defined a novel program slicing algorithm that inspects how the value of a variable is used and changes across recursive calls. We have provided formal definitions for all our concepts. We have implemented our approach in Erlang, producing a parser and prototype classifier. We have applied our prototype to 12 examples, including benchmarks from the NoFib suite and functions from the Haskell Prelude. Our prototype successfully discovers all the maps in our examples. We have also applied our prototype to synthetic benchmarks, showing that our prototype has quadratic time complexity. We have provided speedup results for three of our examples: sumeuler, matrix multiplication, and n-queens. We achieve speedups of up to 32.93 on our 56-core hyperthreaded experimental machine, so demonstrating effectiveness of using map operations as loci of potential parallelism.

For future work, we intend to address the main limitations of our approach, including discovering map operations in functions that traverse multiple data structures of different types. Relatedly, we also intend to expand our technique to inspect how inputs can be divided into sub-groups of inputs that can be performed in parallel, as in, e.g., wavefront parallelism. We intend to expand our property checking to other parallel structures, e.g. divide-andconquer and feedback patterns [9]. Relatedly, as our technique requires any new pattern to be encoded in terms of how values of variables change between recursive calls, we intend to consider how this translation might be simplified. Similarly, we intend to develop the means to translate from full languages, such as Haskell or Erlang, to our expression language. Finally, since our technique is, in principle, language-independent, we also intend to adapt and apply our technique to imperative languages; such as $\mathrm{C}++$ or Java.

\section{References}

[1] Joonseon Ahn and Taisook Han. 2000. An Analytical Method for Parallelization of Recursive Functions. Parallel Processing Letters 10, 1 (2000), 87-98.

[2] Adam D. Barwell, Christopher Brown, David Castro, and Kevin Hammond. 2016. Towards Semi-automatic Data-type Translation for Parallelism in Erlang. In Proceedings of the 15th International Workshop on Erlang (Erlang 2016). 60-61.

[3] Adam D. Barwell, Christopher Brown, Kevin Hammond, Wojciech Turek, and Aleksander Byrski. 2016. Using Program Shaping and Algorithmic Skeletons to Parallelise an Evolutionary Multi-Agent System in Erlang. Computing and Informatics 35, 4 (2016), 792-818.

[4] Adam D. Barwell and Kevin Hammond. 2017. Obliterating Obstructions: Discovering Potential Parallelism by Slicing for Dependencies in Recursive Functions. International fournal of Parallel Programming (2017). In submission. 
[5] Cedric Bastoul. 2004. Code Generation in the Polyhedral Model Is Easier Than You Think. In Proc. of the 13th Intl. Conf. on Parallel Architectures and Compilation Techniques (PACT '04). 7-16.

[6] István Bozó, Viktoria Fordós, Zoltán Horvath, Melinda Tóth, Dániel Horpácsi, Tamás Kozsik, Judit Köszegi, Adam Barwell, Christopher Brown, and Kevin Hammond. 2014. Discovering Parallel Pattern Candidates in Erlang. In Proc. of the 13th ACM SIGPLAN Workshop on Erlang (Erlang '14). 13-23.

[7] Christopher Brown, Marco Danelutto, Kevin Hammond, Peter Kilpatrick, and Archibald Elliott. 2013. Cost-Directed Refactoring for Parallel Erlang Programs. International fournal of Parallel Programming (2013), 1-19.

[8] R. M. Burstall and John Darlington. 1977. A Transformation System for Developing Recursive Programs. F. ACM 24, 1 (Jan. 1977), 44-67.

[9] David Castro, Kevin Hammond, and Susmit Sarkar. 2016. Farms, Pipes, Streams and Reforestation: Reasoning About Structured Parallel Processes Using Types and Hylomorphisms. In Proceedings of the 21st ACM SIGPLAN International Conference on Functional Programming (ICFP 2016). 4-17.

[10] Manuel M.T. Chakravarty, Gabriele Keller, Sean Lee, Trevor L. McDonell, and Vinod Grover. 2011. Accelerating Haskell Array Codes with Multicore GPUs. In Proc. of the 6th Workshop on Declarative Aspects of Multicore Programming. 3-14.

[11] Murray I. Cole. 1988. Algorithmic Skeletons: A Structured Approach to the Management of Parallel Computation. Ph.D. Dissertation.

[12] A. Cook, A. Ireland, G. Michaelson, and N. Scaife. 2005. Discovering Applications of Higher Order Functions Through Proof Planning. Form. Asp. Comput. 17, 1 (May 2005), 38-57.

13] Michael Dever and G. W. Hamilton. 2014. AutoPar: Automatic Parallelization of Functional Programs. In 2014 4th Int. Valentin Turchin Workshop on Metacomputation (META 2014). 11-25

[14] R. Ferenc, A. Beszedes, L. Fulop, and J. Lele. 2005. Design pattern mining enhanced by machine learning. In 21st IEEE International Conference on Software Maintenance (ICSM'05). 295-304.

[15] F. Gava and F. Loulergue. 2005. A static analysis for Bulk Synchronous Parallel ML to avoid parallel nesting. Future Generation Computer Systems 21, 5 (2005), $665-671$.

[16] Louis Gesbert, Frdric Gava, Frdric Loulergue, and Frdric Dabrowski. 2010. Bulk synchronous parallel ML with exceptions. Future Generation Computer Systems $26,3(2010), 486-490$.

[17] Alfons Geser and Sergei Gorlatch. 1999. Parallelizing Functional Programs by Generalization. f. Functional Programming 9, 06 (1999), 649-673.

[18] Jeremy Gibbons. 1996. The Third Homomorphism Theorem. fournal of Functional Programming (FFP) 6, 4 (1996), 657-665.

[19] Sergei Gorlatch. 1999. Extracting and Implementing List Homomorphisms in Parallel Program Development. Science of Computer Programming 33, 1 (1999).

[20] J. C. Guzman and P. Hudak. 1990. Single-threaded polymorphic lambda calculus. In Logic in Computer Science. 333-343.

[21] Clemens Hammacher, Kevin Streit, Sebastian Hack, and Andreas Zeller. 2009 Profiling Java Programs for Parallelism. In Proceedings of the 2009 ICSE Workshop on Multicore Software Engineering (IWMSE '09). 49-55.

[22] Kevin Hammond, Marco Aldinucci, Christopher Brown, Francesco Cesarini, Marco Danelutto, Horacio González-Vélez, Peter Kilpatrick, Rainer Keller Michael Rossbory, and Gilad Shainer. 2013. The ParaPhrase Project: Parallel Patterns for Adaptive Heterogeneous Multicore Systems. In Formal Methods for Components and Objects. Lecture Notes in Computer Science, Vol. 7542. Springer Berlin Heidelberg, 218-236.

[23] Steve Holzner. 2004. Eclipse: A Java Developer's Guide. O'Reilly \& Associates, Inc., Sebastopol, CA, USA.

[24] Zhenjiang Hu, Masato Takeichi, and Hideya Iwasaki. 1999. Diffusion: Calculating Efficient Parallel Programs. In Proc. PEPM'99. 85-94.

[25] V. Janjic, C. Brown, and K. Hammond. 2016. Lapedo: Hybrid Skeletons for Programming Heterogeneous Multicore Machines in Erlang. In Proc. ParCo 2015: Intl. Conf. on Parallel Computing. 181-195.

[26] Venkatesh Kannan and Geoff W. Hamilton. 2016. Program Transformation to Identify Parallel Skeletons. In 24th Euromicro International Conference on Parallel, Distributed, and Network-Based Processing, PDP 2016, Heraklion, Crete, Greece, February 17-19, 2016. 486-494.

[27] Huiqing Li and Simon Thompson. 2015. Safe Concurrency Introduction Through Slicing. In PEPM '15. 103-113.

[28] Huiqing Li, Simon J. Thompson, George Orösz, and Melinda Tóth. 2008. Refactoring with wrangler, updated: data and process refactorings, and integration with eclipse. In Proc. of the 7th ACM SIGPLAN Workshop on Erlang. 61-72.

[29] Simon Marlow, Patrick Maier, Hans-Wolfgang Loidl, Mustafa K. Aswad, and Phil Trinder. 2010. Seq No More: Better Strategies for Parallel Haskell. In Proc. of the 3rd ACM Haskell Symp. on Haskell (Haskell '10). 91-102.

[30] Simon Marlow, Ryan Newton, and Simon Peyton Jones. 2011. A Monad for Deterministic Parallelism. In Proc. of the 4th ACM Symposium on Haskell (Haskell '11). 71-82.

[31] Erik Meijer, Maarten Fokkinga, and Ross Paterson. 1991. Functional Programming with Bananas, Lenses, Envelopes and Barbed Wire. In Proceedings of the 5th ACM Conference on Functional Programming Languages and Computer Architecture. $124-144$.

[32] Will Partain. 1993. The nofib Benchmark Suite of Haskell Programs. In Proc. of the 1992 Glasgow Workshop on Functional Programming, John Launchbury and
Patrick Sansom (Eds.).

[33] Benjamin C. Pierce. 2004. Advanced Topics in Types and Programming Languages. The MIT Press.

[34] Martin P. Robillard. 2008. Topology Analysis of Software Dependencies. ACM Trans. Softw. Eng. Methodol. 17, 4, Article 18 (Aug. 2008), 36 pages.

[35] Norman Scaife, Susumi Horiguchi, Greg Michaelson, and Paul Bristow. 2005. A parallel SML compiler based on algorithmic skeletons. Fournal of Functional Programming 15, 4 (2005), 615-650.

[36] Temple F Smith and Michael S Waterman. 1981. Identification of common molecular subsequences. Fournal of molecular biology 147, 1 (1981), 195-197.

[37] P. W. Trinder, K. Hammond, H.-W. Loidl, and S. L. Peyton Jones. 1998. Algorithm + Strategy = Parallelism. f. Funct. Program. 8, 1 (Jan. 1998), 23-60.

[38] Mark Weiser. 1981. Program Slicing. In Proc. of the 5th Intl. Conf. on Software Engineering (ICSE '81). 439-449. 